\title{
The Propagation of Monochromatic Electromagnetic Radiation Inside of Luneburg Lens in Relative Coordinate Frame of Reference
}

\author{
Gladyshev V. O. ${ }^{1}$, Tereshin A. A. ${ }^{1}$, Yavorskiy A. V. ${ }^{2}$, and Bazleva D. D. ${ }^{1}$ \\ ${ }^{1}$ Bauman Moscow State Technical University, Moscow, 105005, Russia \\ ${ }^{2}$ Open Joint-Stock Company "Research Institute of Precision Instruments", Moscow, 127490, Russia
}

Keywords: Luneburg lens, gradient lenses, the dispersion equation, satellite reflector, angular deviation.

\begin{abstract}
A mathematical model of the propagation of laser radiation inside Luneburg lens in relative coordinate frame of reference has been developed. The trajectory of the beam propagation inside the lens, as well as angles of incidence, refraction and reflection have been defined. In addition to this on the basis of the data received we plotted some charts with the help of which one could determine the amount of the aberration of the beam, operation region of the lens, deviation of the beam from the detector after its propagation in the lens. It was shown that the deviation of the beam in the plane of the registration on the ground does not exceed 200 meters.
\end{abstract}

\section{Introduction}

Recently a new type of gradient lenses - Luneburg lens has been widely adopted. The index of refraction in this lens is not permanent changing from point to point. The law of variation of the index of refraction is usually chosen in such a way that incoming parallel beams after their propagation through the lens are focused on the other side of the lens in one point. Then the reflected beams form beams parallel to the incident ones.

Luneburg lenses are usually applied in radio-location. It was used for the first time in the American radar AN/SPG-59 as a beam shaper at the beginning of 1960s and after it was widely adopted.

In the work [1] authors described a scheme of the antenna, in which Luneburg lens of different forms could be used (spherical, cylindrical, multy-layer were calculated by their mathematical model).

In another work [2] was offered an integrated design of Luneburg lens, which was made of silicone with ultra-high gradient index. Such lens will have low losses, high index contrast and with the help of integrated nanophotonic waveguides one could obtain high-precision connection between the fibre and the chip.

In the patent [3] authors described a design of the lens reflector with cross polarization. Luneburg lens was used as a reflector with spherically imperfect reflection surface, which consists of conductive stripes placed at an angle $45^{\circ}$ to the polarization of the incident wave.

In the optical range Luneburg lens replaces triple prisms, which are used as angular reflectors on the board of up-to-date satellite navigation systems such as GLONASS, GPS and GALILEO. To achieve large effective area of reflection of the incident wave it is required a matrix consist of large amount of the angular reflectors.

If to use Luneburg lens as a reflector, the power of reflected radiation will be compared to the power of the reflected radiation obtained by a similar matrix of angle reflectors. It is worth noting that the calculation of such lens is usually made without taking into account of the effects of the optics of moving media. These effects could exercise a significant influence on the accuracy of the determination of the spatial-temporal coordinates of the spacecraft. 


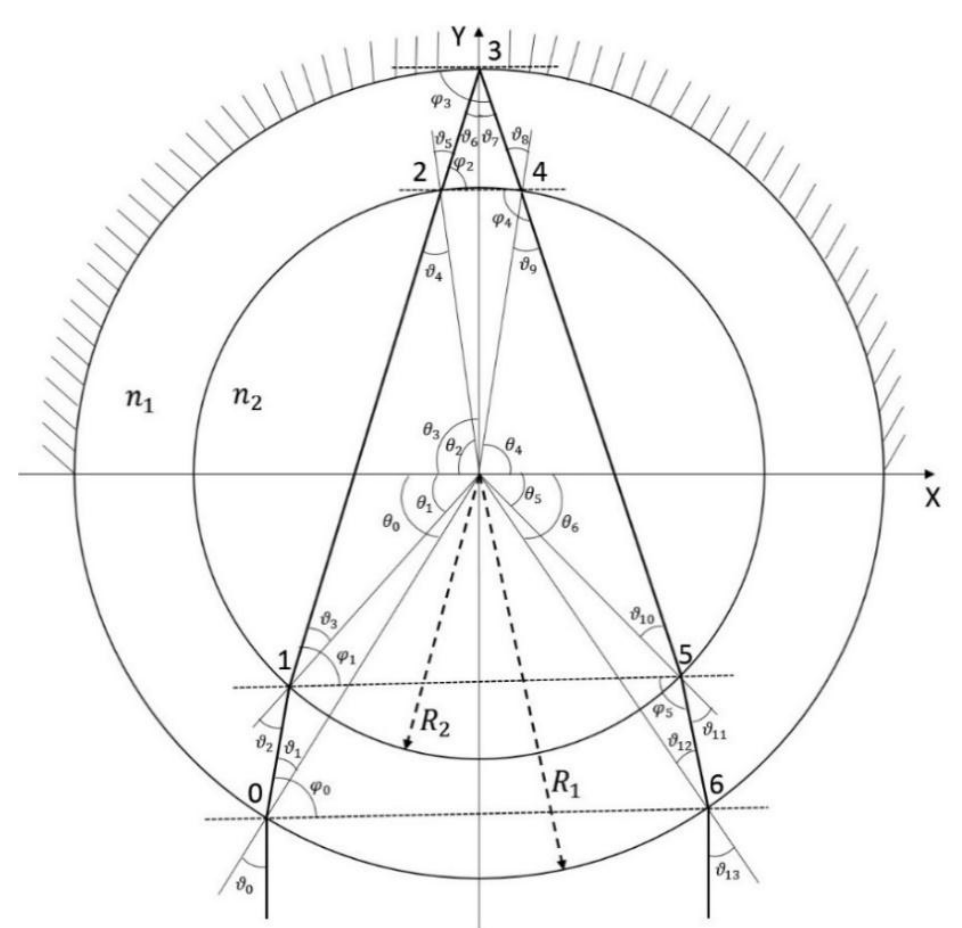

Fig. 1. The trajectory of the beam inside of the lens.

\section{Statement of the Problem}

In this article we introduce the algorithm of calculation of the beam propagation in the Luneburg lens, which was used on board nanosatellite BLIZ [4]. This satellite was represented as an autonomous spherical glass laser system, which was located at geosynchronous solar orbit for 3 years. Different scientific problems connected with geophysics, geodynamics and theory of relativity were investigated with the help of this system. It was also used for solving different problems of high precision calculations and long-term forecasting of orbits of specialized geodetic navigation space vehicles.

The orbital altitude of this satellite was $\mathrm{h}=835 \mathrm{~km}$. The Luneburg lens, which was used on board of this satellite, consisted of two layers (Fig. 1). The radius of the central orb was $R_{2}=53.5 \mathrm{~mm}$ and it was made of heavy flint with the index of refraction $n_{2}=1.7647$. The radius of the external meniscus was $R_{1}=85 \mathrm{~mm}$ and it was made of light crown with the index of refraction $n_{1}=1.4729$. One half of the external meniscus was mirrored. The speed of rotation around the axis perpendicular to the orbit plane of this satellite was 10 rotations per minute. The wavelength of the laser radiation was $\lambda=532 \mathrm{~nm}$.

It was necessary to calculate 13 angles and trajectory of the laser beam inside of The beams were falling from the right as well as from the left side of the lens. the lens according to Fig. 1 taking into account the trajectory of the satellite. The beams were falling from the right as well as from the left side of the lens.

\section{The Description of the Propagation of Electromagnetic Radiation Inside of Luneburg Lens}

We used the solution of the dispersion equation which was obtained by Bolotovsky B. M. and Stoliarov S. N. [5] and was checked in [6,7].

In case if the falling wave is specified, the tangential component of the wave vector can be defined as $\vec{k}_{t}=\vec{I}_{t}$ and the following expression is true $I_{1}=k_{n} v-w_{e}$, where $k_{n}=(\vec{k}, \vec{v}), \vec{n}-$ normal unit vector to the boundary, which is directed from the first medium to the second one.

$$
\left(w_{1}\right)_{1,2}=-I_{1} \frac{\left[1+\kappa_{1} \gamma_{1}^{2}\left(\beta-\beta_{1 n}\right)\left(\beta_{1 n}+\left(\vec{\beta}_{1 t}, \vec{d}\right) \beta\right)\right] \pm \beta Q_{1}^{\frac{1}{2}}}{\left(1-\beta^{2}\right)-\kappa_{1} \gamma_{1}^{2}\left(\beta-\beta_{1 n}\right)^{2}},
$$




$$
\begin{array}{rl}
Q_{1}= & {\left[1+\kappa_{1} \gamma_{1}^{2}\left(1-\beta_{1 n}^{2}\right)\right]-} \\
& -\vec{d}^{2}\left[\left(1-\beta^{2}\right)-\kappa_{1} \gamma_{1}^{2}\left(\beta-\beta_{1 n}\right)^{2}\right]+ \\
& +\kappa_{1} \gamma_{1}^{2}\left(\vec{\beta}_{1 t}, \vec{d}\right)\left[2\left(1-\beta \beta_{1 n}\right)+\left(1-\beta^{2}\right)\left(\vec{\beta}_{1 t}, \vec{d}\right)\right], \\
d=c & c \frac{I_{t}}{I_{1}}, \quad \kappa_{1}=\varepsilon_{1} \mu_{1}-1, \quad \beta=\frac{v}{c}, \quad \beta_{1 n}=\frac{u_{1 n}}{c}, \\
\beta_{1 t}= & \frac{u_{1 t}}{c}, \quad \gamma_{1}^{2}=\frac{1}{1-\beta_{1}^{2}}, \quad \beta_{1}^{2}=\frac{u_{1}^{2}}{c^{2}}=\beta_{1 n}^{2}+\beta_{1 t}^{2},
\end{array}
$$

$u_{1 n}$ and $u_{1 t}$ are normal and tangential components of the speed of one medium to the interface. From the formula (1) we can obtain two values for the frequency in the first medium, which are expressed in terms of invariants $I_{1}$ and $\vec{I}_{t}$, parameters of the medium $\left(\varepsilon_{1}, \mu_{1}, u_{1}\right)$ and velocity $\vec{v}$ of the interface. One of the obtained values defines the frequency of the incident wave and the other one - the frequency of the reflected wave. If the frequencies $\left(w_{1}\right)_{1,2}$ are known, then we can obtain the corresponding values for the normal component of the wave vector

$$
\begin{aligned}
\left(k_{1 n}\right)_{1,2} & =\left[\left(w_{1}\right)_{1,2}+I_{1}\right] v^{-1}= \\
& =-I_{1} \frac{\left[\beta+\kappa_{1} \gamma_{1}^{2}\left(\beta-\beta_{1 n}\right)\left(1+\left(\vec{\beta}_{1} t, \vec{d}\right)\right)\right] \pm Q_{1}^{\frac{1}{2}}}{c\left[\left(1-\beta^{2}\right)-\kappa_{1} \gamma_{1}^{2}\left(\beta-\beta_{1 n}\right)^{2}\right]}
\end{aligned}
$$

We can also write a similar expression for the wave vector for the second medium.

One should keep in mind the fact that when light is passing through a medium the following parameters will be changing: frequency, wave vector and speed of light. The new value of the wave vector will be defined as

$$
k_{i}=\sqrt{k_{n i}^{2}+k_{t i}^{2}}
$$

The new value of the frequency will be calculated from

$$
w_{i}=k_{n i} \beta c_{0}+I_{1} \text {, }
$$

where $c_{0}$ is the speed of light in vacuum.

The new value of the speed of light will be as follows

$$
c_{i}=\frac{w_{i} \cos \left(\vartheta_{i}\right)}{\left|k_{n i}\right|},
$$

where $i=0,1,2, \ldots, 6$.

To define the coordinates of the $i$ - point we need to write the equation of the circle

$$
\left(x_{k}-v_{x}\left(t_{k}+T\right)\right)^{2}+\left(y_{k}-v_{y}\left(t_{k}+T\right)\right)^{2}=R_{1.2}^{2}, \quad k=1,2, \ldots, 6
$$

and the equation of the beam in parametric form

$$
\left\{\begin{array}{l}
x_{k}=x_{k-1} \pm c_{i} t_{k} \cos \left(\varphi_{i}\right) \\
y_{k}=y_{k-1} \pm c_{i} t_{k} \sin \left(\varphi_{i}\right)
\end{array}\right.
$$

where $\varphi_{i}-$ is some auxiliary angle, the calculation of which will be shown below;

$T=\sum_{k=1}^{i} t_{k-1}-$ is the total value of time, during which the center of the lens is moved when considering the point $k$.

The sign in equation (7) will be chosen considering the beam before reflection (" + ") in point 3 or after it ("-"). 
To determine the time of beam propagation between two points we need to substitute equation (7) in (6) and solve it with relation to time

$a_{1,2} t^{2}+b_{1,2} t+c=0$,

where in case of beam propagation in the interval 1-3 before its reflection in point 3 the coefficients are

$$
\begin{aligned}
\mathrm{a}_{1}= & \left(\mathrm{c} \sin \left(\varphi_{i}\right)-v_{y}\right)^{2}+\left(\mathrm{c} \cos \left(\varphi_{i}\right)-v_{x}\right)^{2} \\
b_{1}= & 2\left(-T v_{x}+x_{k-1}\right)\left(\mathrm{c} \cos \left(\varphi_{i}\right)-v_{x}\right)+ \\
& +2\left(-T v_{y}+y_{k-1}\right)\left(\mathrm{c} \sin \left(\varphi_{i}\right)-v_{y}\right)
\end{aligned}
$$

We can write down the same expressions for $a_{2}$ and $b_{2}$ in the interval of points 3-6 after the reflection of the beam, but we should take into account that the sign before coefficient $c$ will be changed to "-".

Coefficient $c$ for both cases will be the same

$$
\mathrm{c}=\left(T v_{x}+x_{k-1}\right)^{2}+\left(T v_{y}+y_{k-1}\right)^{2}-R_{1,2}^{2},
$$

where $R_{1,2}$ - are radii of the outer meniscus and inner orb; the selection of the radius depends on the intersection point of the beam with the lens.

Then the desired solution of the equation (8) according to the beam propagation in the interval of points from 0 to 3 before its reflection in point 3 will be as follows

$$
\begin{aligned}
t_{1.2}= & \frac{-2\left(-T v_{x}+x_{k-1}\right)\left(\mathrm{c} \cos \left(\varphi_{i}\right)-v_{x}\right)}{2\left(\left(\mathrm{c} \sin \left(\varphi_{i}\right)-v_{y}\right)^{2}+\left(\mathrm{c} \cos \left(\varphi_{i}\right)-v_{x}\right)^{2}\right)}- \\
& -\frac{2\left(-T v_{y}+y_{k-1}\right)\left(\mathrm{c} \sin \left(\varphi_{i}\right)-v_{y}\right)}{2\left(\left(\mathrm{c} \sin \left(\varphi_{i}\right)-v_{y}\right)^{2}+\left(\mathrm{c} \cos \left(\varphi_{i}\right)-v_{x}\right)^{2}\right)} \pm \\
& \pm \frac{\sqrt{b_{1}^{2}-4 \mathrm{a}_{1} c}}{2\left(\left(\mathrm{c} \sin \left(\varphi_{i}\right)-v_{y}\right)^{2}+\left(\mathrm{c} \cos \left(\varphi_{i}\right)-v_{x}\right)^{2}\right)}
\end{aligned}
$$

In case the beam is reflected from the inner-mirrored side of the meniscus in point 3 , we will obtain the same expression but the sign before coefficient $c$ will be changed to the "-" except for $\sqrt{b_{2}^{2}-4 \mathrm{a}_{2} c}$.

The two values of time determined from expression (11) are substituted in (7) yielding two pairs of coordinates $(x, y)$. After this we should choose the right one in accordance with the geometry of the problem being solved.

To evaluate the angle between the normal and the falling beam for every point we should write the equation of the held normal to the circle (Fig. 1) [8]

$$
y-y_{0}=-\frac{1}{y^{\prime}\left(x_{0}\right)}\left(x-x_{0}\right),
$$

where $y^{\prime}\left(x_{0}\right)$ is the differential quotient of the equation of the curve at the point of observation.

With evaluated $y^{\prime}\left(x_{0}\right)$, we need to reduce equations (12) and (7) to canonical form

$$
\begin{aligned}
& \frac{y-y_{0}}{y_{0}-v_{y}\left(t_{k}+T\right)}=\frac{x-x_{0}}{x_{0}-v_{x}\left(t_{k}+T\right)} \\
& \frac{y-y_{0}}{\operatorname{csin}\left(\varphi_{i}\right)}=\frac{x-x_{0}}{\cos \left(\varphi_{i}\right)}
\end{aligned}
$$

Taking into account these two equations (13) and (14) we can define the desired angle $\vartheta$ 


$$
\cos (\vartheta)=\frac{\left|\left(x_{0}-v_{x}\left(t_{k}+T\right)\right) \cos \left(\varphi_{i}\right)+\left(y_{0}-v_{y}\left(t_{k}+T\right)\right) c \sin \left(\varphi_{i}\right)\right|}{c_{i} \sqrt{\left(x_{0}-v_{x}\left(t_{k}+T\right)\right)^{2}+\left(y_{0}-v_{y}\left(t_{k}+T\right)\right)^{2}}}
$$

Then we should calculate auxiliary angles $\varphi_{j}$. For this purpose, we need to write down the equation of the held normal at $i$-point and the equation of the beam, crossing this point

$$
\begin{aligned}
& y=\operatorname{tg}\left(\varphi_{j}\right) x+\left(y_{0}-\operatorname{tg}\left(\varphi_{j}\right) x_{0}\right) \\
& y=\frac{y_{0}-v_{y}\left(t_{k}+T\right)}{x_{0}-v_{x}\left(t_{k}+T\right)} x-\left(\frac{y_{0}-v_{y}\left(t_{k}+T\right)}{x_{0}-v_{x}\left(t_{k}+T\right)} x_{0}-y_{0}\right)
\end{aligned}
$$

The slope coefficients of the normal and the beam will be determined as

$$
s_{1}=\frac{y_{0}-v_{y}\left(t_{k}+T\right)}{x_{0}-v_{x}\left(t_{k}+T\right)}, s_{2}=\operatorname{tg}\left(\varphi_{j}\right), j=0, \ldots, 5
$$

The angle between two lines can be defined as [8]

$$
\operatorname{tg}\left(\vartheta_{i+1}\right)=\frac{s_{1}-s_{2}}{1+s_{1} s_{2}}, \quad i=0, \ldots, 10
$$

As the angle $\vartheta_{i+1}$ is known, we can find the expression for the auxiliary angle $\varphi_{j}$ from the equation (19)

$$
\varphi_{j}=\arctan \left(\frac{\operatorname{tg}\left(\vartheta_{i+1}\right)\left(t_{k}+T\right) v_{x}-\operatorname{tg}\left(\vartheta_{i+1}\right) x+\left(t_{k}+T\right) v_{y}-y}{\operatorname{tg}\left(\vartheta_{i+1}\right)\left(t_{k}+T\right) v_{y}-\operatorname{tg}\left(\vartheta_{i+1}\right) y-\left(t_{k}+T\right) v_{x}+x}\right)
$$

And the last step will be the determination of the slope of the normal to the $O x$ axe. This angle is essential for the determining of the deviation of the beam at the output of the lens in accordance with the incident beam.

To calculate this angle we need to take the arctangent from $s_{1}$

$$
\theta_{m}=\arctan \left(s_{1}\right)=\arctan \left(\frac{y_{0}-v_{y}\left(t_{k}+T\right)}{x_{0}-v_{x}\left(t_{k}+T\right)}\right), \quad m=0 \ldots 6
$$

After this we need to compare two values $d \vartheta_{0}, d \vartheta$, which will be calculated according to the following expressions (Fig. 1)

$$
d \vartheta_{0}=\left|\vartheta_{0}+\theta_{0}\right|-\left|\vartheta_{13}+\theta_{6}\right| ; d \vartheta=\left|\vartheta_{0}+\theta_{0}\right|-\left|\vartheta_{13}+\theta_{6}\right|,
$$

where $d \vartheta_{0}$ is the difference between the sum of the incident angle $\vartheta_{0}$ at the point where the beam enters the lens, and the angle $\theta_{0}$ defining the normal at point 0 , and the sum of the refraction angle $\vartheta_{13}$ at the point where the beam left the lens, and the angle $\theta_{6}$ defining the normal at the point 6 (the lens does not have speed);

$d \vartheta$ is the difference between the sum of the incident angle $\vartheta_{0}$ at the point where the beam enters the lens, and the angle $\theta_{0}$ defining the normal at the 0 point, and the sum of the refraction angle $\vartheta_{13}$ at the point where the beam left the lens, and the angle $\theta_{6}$ defining the normal at point 6 (the lens have the speed of $7500 \mathrm{~m} / \mathrm{c}$ );

If we evaluated $d \vartheta_{0}, d \vartheta$ previously, we can define the deviation of the beam relative to the detector after its reflection by the Luneburg lens in accordance with its movement speed

$$
\begin{aligned}
& d l_{0}=\operatorname{tg}\left(d \vartheta_{0}\right) h \\
& d l=\operatorname{tg}(\mathrm{d} \vartheta) h,
\end{aligned}
$$


where $d l_{0}$ is the distance of the beam deviation from the emitter after its reflection by the lens and return to the ground in case the lens does not move;

$d l$ is the distance of the beam deviation from the emitter after its reflection by the lens and return to the ground in case the lens moves with the speed of $7500 \mathrm{~m} / \mathrm{c}$;

Analogous calculations were made for the case when the beam was falling from the right side.

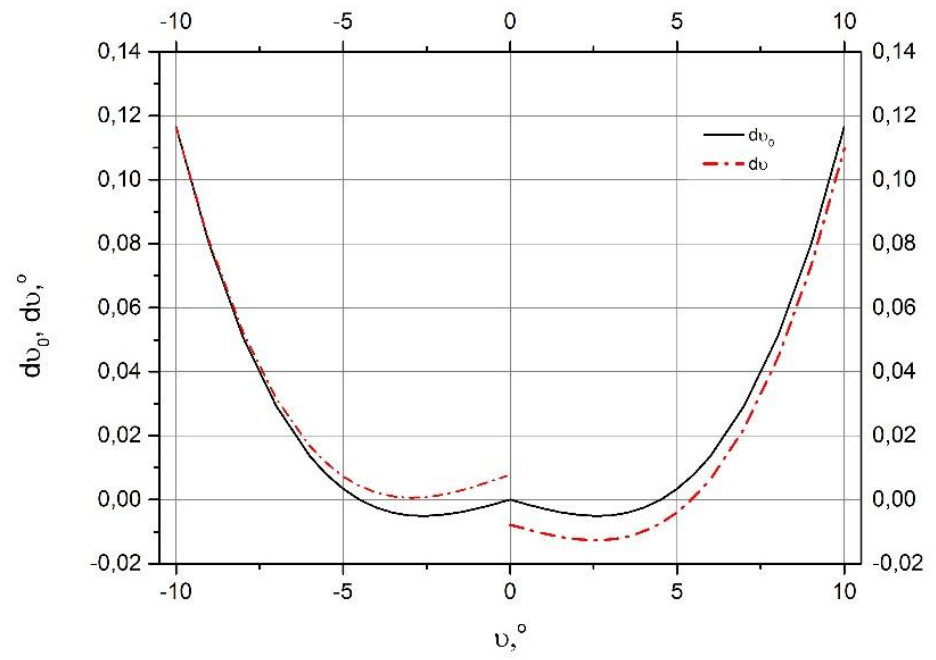

Fig. 2. $d \vartheta_{0}$ и $d \vartheta$ dependence on the incident angle $\vartheta\left(d \vartheta_{0}(\vartheta)-\right.$ is the graphic plotted for the case when the lens have a speed; $d \vartheta(\vartheta)$ - is the graphic plotted for the case when the lens is rested).

\section{Numerical Data}

On the basis of the received data we plotted the following charts: $d \vartheta_{0}(\vartheta), d \vartheta(\vartheta)$ (Fig. 2).

In Figure 2 we introduce dependences $d \vartheta_{0}(\vartheta), d \vartheta(\vartheta)$, which are up to the incident angle $\vartheta$ with and without the speed of the lens. If we look at the obtained graphs, we will notice the interval of incident angles in which $d \vartheta_{0}$ and $d \vartheta$ are negative. It can be explained by small oscillations of coordinates of point 3 . This leads to the offset of points 4, 5, 6 .

According to the expression (21) when we determine the angle $\theta_{m}$ we calculate the arctangent of the slope coefficient of the normal $s_{1}$. This coefficient depends on the values of the coordinates, which we calculated earlier. The value of the normal angle will be slightly different from the normal angle at the entrance point of the beam. If we compare the coordinates of the entrance of the beam and coordinates of its exit from the lens, we will notice that the exiting coordinates are slightly different from the entrance coordinates. Because of this difference, there appeared a negative area on the graphs.

Starting with some value of the incident angle, $d \vartheta_{0}$ and $d \vartheta$ begin to increase dramatically. This area is not so interesting, because of big values of incident angles, which cause big values for the deviation of the beam relative to the detector after its reflection by the lens.

The presence of the speed of the lens leads to the symmetry breakdown of the beam propagation inside the lens which is observed when the lens is at rest.

With the help of the calculated data and formulas (23-24) we determine the cross-section of the beam returning to the ground. In the interval of angles of incidence from -2.5 to -1.5 and from 1.5 to 2.5 we obtained the minimum deviation of the beam returned to the ground and it was not more than 100 meters.

\section{Conclusions}

We plotted the following charts: $d \vartheta_{0}(\vartheta), d \vartheta(\vartheta)$. If we analyze the graphs shown in the figure, we can determine the interval of angles (from -2.5 to -1.5 and from 1.5 to 2.5 ) in which the intensity of the 
beam registered by the detector will be maximum. It is also possible to obtain some estimations of the cross-section of the beam returning to the ground.

From Figure 2 it is possible to define the amount of aberration of the beam after its passing through the lens and to see the area in which this value will be minimum.

It is very important to take into account effects of the optics of the movable medium, while evaluating such systems. The calculation of the lens is shown that effects cause the deviation of the beam in the zone of detection for about hundreds of meters, which is essential in defining time and cords of the satellites, such as GLONASS. We are planning to expand our mathematical model and take into account effects of polarization of the radiation $[9,10]$, relativistic effects etc.

\section{References}

[1] Slavi Baev, Boyan Hadjistamov, Plamen Dankov, Luneberg lenses as communication antennas, St. Kliment Ohridski University of Sofia, Faculty of Physics, 2009, p. 18.

[2] Jonathan Bor, Olivier Lafond, Herve Merlet, Philippe Le Bars, Mohamed Himdi, foam Based Luneberg Lens Anenna at 60 GHz, Progress In electromagnetics Research Letters, Vol.44, 2014, pp. 1-7.

[3] Patent 3972043 Northrop Corporation, Cross-polarizing lens reflector / Silvan Stanley Locus. Published 27.07.1976.

[4] Chazov V. V., Development and application of the algorithms of the numeric calculation of the satellite position. LMSU, Moscow, 2012,p. 156.

[5] Bolotovskiy B. M., Stoliarov S. H., The reflection of the light from the moving mirror and kindred problems. UFN. -1989. - T. 159. - pp. 155-180.

[6] Gladyshev V. O.,Gladysheva T. M., Zubarev V. E., The Effect of Light Entrainment Observed in an Optical Disk Interferometer, Technical Physics Letters, vol. 28, №2, p. 123.

[7] Gladyshev V. O., Gladysheva T. M., Dashko M. I., Trofimov N. E., and Sharandin E. A., First Results of Measurements of the Rotation Speed Effecton the Spatial Entrainment of Light in a Rotating Medium. Technical Physics Letters, vol. 33, №11, p. 905.

[8] Korn G., Korn T., Handbook of Mathematics for Scientific Workers and Engineers. - M.: Science, 1984. - p. 831.

[9] Gladyshev V. O., Portnov D. I., Kautz V. L., and Sharandin E. A., Experimental Studies of Polarization of Laser Radiation in a Rotating Optical Glass. Optics and Spectroscopy, vol. 115, № 3б 2013. p. 349.

[10] Gladyshev V. O., Portnov D. I., Evolution of the Polarization of the He-Ne Laser Radiation in Rotating Dielectric. Technical Physics, vol. 85, № 4, 2015. pp. 97-104. 